\title{
Migración infrecuente de tuberculosis extrapulmonar primaria: reporte de caso
}

Marcia Darmelly Salas

Pérez

ORCID: 0000-0002-6398-2369

Médica cirujana. Especialidad en

Medicina Interna. Hospital Nacional

Dos de Mayo. Universidad Nacional Mayor de San Marcos.

\section{Oscar Orlando Rivera}

Torrejón

ORCID: 0000-0001-8524-0553

Estudiante de Medicina Humana.

Universidad Nacional Mayor de San

Marcos. Sociedad Científica de San

Fernando - SCSF.

Diego Chambergo Michilot

ORCID: 0000-0002-3904-7514

Estudiante de Medicina Humana.

Facultad de Ciencias de la Salud.

Universidad Científica del Sur.

Sociedad Científica de Estudiantes

de Medicina de la Universidad

Científica del Sur - SCIEM-UCSUR.

Ubaldo Paucar Pongo

ORCID: 0000-0002-4130-3118

Estudiante de Medicina Humana. Universidad Nacional de San Agustín

de Arequipa.

Sociedad Científica de Estudiantes

de Medicina Agustinos - SOCIEMA.

\author{
Infrequent migration of primary extrapulmonary tuberculosis: case report \\ Migração infreqüente de tuberculose extrapulmonar primária: relato de \\ caso
}

Resumen: A nivel global, la tuberculosis de localización extrapulmonar representa el $18 \%$ de los casos de la enfermedad, teniendo como lugares más frecuentes de afectación la pleura (54\%), ganglios $(11,1 \%)$, sistema nervioso central $(9 \%)$ y sistema osteoarticular (3,6\%), entre otros. La manifestación clínica de la meningoencefalitis tuberculosa se presenta con fiebre, cefalea, vómitos, alteración de la conciencia, fotofobia, afectación de pares craneales, alteraciones audiovisuales, signos de irritación meníngea y focalización neurológica. La tuberculosis ostearticular es consecuenciadeunadiseminaciónhemática, linfáticao, excepcionalmente, porinoculacióndirecta. La clínica es insidiosa, con dolor, inflamación y disminución del rango articular, pudiendo presentar abscesos y cavidades supurativas. Sin embargo, existen otros síntomas de baja frecuencia de aparición, dificultando el diagnóstico adecuado. Se describe el caso clínico infrecuente de tuberculosis extrapulmonar, destacando la diseminación de Mycobacterium tuberculosis con foco en la articulación coxofemoral izquierda hacia meninges y cerebro, y el uso inapropiado del tratamiento con corticoesteroides en un paciente seronegativo para $\mathrm{VIH}$.

Palabras clave: tuberculosis, meningoencefalitis, tuberculosis osteoarticular, glucocorticoides, dexametasona.

Abstract: Extrapulmonary tuberculosis accounts for $18 \%$ of tuberculosis cases, with the pleura (54\%), lymph nodes (11.1\%), central nervous system (9\%) and osteoarticular system (3.6\%) as sites of involvement, among others. Clinical manifestations of tuberculous meningoencephalitis are fever, headache, vomiting, altered consciousness, photophobia, cranial nerve involvement, audiovisual alterations, signs of meningeal irritation and neurological focalization. Ostearticular tuberculosis is the result of hematic, lymphatic dissemination or, exceptionally, by direct inoculation. The clinic is insidious, with pain, inflammation and diminished joint range, and can present abscesses and suppurative cavities. However, there are other symptoms of low frequency of appearance, making the diagnosis difficult. For this reason, an infrequent clinical case of extrapulmonary tuberculosis is described, highlighting the spread of Mycobacterium tuberculosis with focus on the left coxofemoral joint to the meninges and brain, and the inappropriate use of adrenal cortex hormones therapy in a seronegative patient for HIV.

Key words: tuberculosis, meningoencephalitis, osteoarticular, glucocorticoids, dexamethasone

Resumo: A tuberculose extrapulmonar é responsável por $18 \%$ dos casos de tuberculose, com pleura (54\%), linfonodos $(11,1 \%)$, sistema nervoso central $(9 \%)$ e sistema osteoarticular $(3,6 \%)$ como locais de envolvimento. entre outros. A manifestação clínica da meningoencefalite tuberculosa é febre, dor de cabeça, vômitos, consciência alterada, fotofobia, comprometimento dos nervos cranianos, alteraçõesaudiovisuais, sinais de irritação meníngea e focalização neurológica. A tuberculose ostearticular é o resultado de disseminação hemática, hemática ou, excepcionalmente, por inoculaçãodireta. A clínica é insidiosa, comdor, inflamação e diminuição do leque de articulações, podendoapresentarabscessos e cavidades supurativas. No entanto, existemoutrossintomas de baixafrequência de aparecimento, dificultando o diagnóstico. Por esta 
razão, nósdescrevemosum caso clínico raro de tuberculoseextrapulmonar, com destaque para a disseminação de Mycobacterium tuberculosis com foco naarticulação do quadrilesquerdo para meninges e cérebro, e uso inadequado de corticoterapiaem paciente soronegativa HIV.

Palavras-chave: tuberculose, meningoencefalite, tuberculoseosteoarticular, glicocorticóides, dexametasona 


\section{Introducción}

La tuberculosis (TBC) afecta entre un 10 a $20 \%$ a órganos extrapulmonares, siendo más afectadas las personas inmunodeprimidas. La presentación extrapulmonar puede ser primaria; corresponder a una diseminación linfohemática, generalmente desde un foco pulmonar; o excepcionalmente, por inoculación directa. En Perú, 31 mil casos nuevos fueron diagnosticados en 2010 , entre 2013 y 2014 se reportaron casi un $18 \%$ de casos de TBC extrapulmonar, el mayor porcentaje de casos presentó localización pleural (54\%), seguida de ganglionar $(11,1 \%)$, del sistema nervioso central (SNC 9\%) y osteoarticular $(3,2 \%)^{(1,2)}$.

La meningitis tuberculosa (MTBC) es la forma clínica más grave, la cual representa el $1 \%$ de los casos de TBC globalmente ${ }^{(3,4)}$. Un estudio en España que evaluó pacientes con MTBC entre 1992 y 2007 halló que el $71 \%$ pertenecían al medio rural, y el 57\% del total eran niños menores de 5 años ${ }^{(5)}$. La presentación clínica de la meningitis abarca fiebre, cefalea, fotofobia, signos de irritación meníngea, rigidez de nuca, signos de Brudzinski y Kernig; y la meningoencefalitis agrega vómitos, afectación de pares craneales, alteración del sensorio, focalización neurológica, y alteraciones audiovisuales ${ }^{(3,5,6)}$. El diagnóstico de laboratorio es por análisis del líquido cefalorraquídeo (LCR) mediante pruebas directas que detectan componentes del microorganismo en este, e indirectas como detección de adenosíndesaminasa (ADA), que se basan en la respuesta inmune del huésped al Mycobacterium tuberculosis o BK ${ }^{(3)}$.

La presentación inicial de la tuberculosis osteoarticular (TBCOA) suele ser una osteomielitis metafisiaria que se extiende a espacios articulares, se describe como monoartritis con preferencia por articulaciones que soportan peso, como la cadera, vértebras y rodillas ${ }^{(7)}$. La presentación de la TBC de cadera es granular y/o exudativa. Esta última predomina en inmunodeprimidos. Se describe la coexistencia de ambos tipos con la predominancia de uno. La forma granular es menos agresiva y presenta erosiones subcondrales; la exudativa es más agresiva, presentando destrucción del hueso y formación de cáseum ${ }^{(8)}$. Cabe mencionar que en un $50 \%$ de los casos de TBCOA, no se aprecian hallazgos radiográficos torácicos ${ }^{(9)}$.

\section{Caso clínico}

Varón mestizo de 33 años, originario de Huánuco (Perú), de ocupación chofer interprovincial durante los últimos 10 años. No cuenta con carné de inmunizaciones, VIH negativo, no refiere alergias ni hábitos nocivos. Sin antecedentes personales ni familiares patológicos. Sin antecedentes quirúrgicos.

En diciembre del 2017, el paciente acudió a emergencias por cefalea holocraneana de intensidad 9/10, vómitos, escalofríos y fiebre. Al examen general se evidenció fascies cushingoides. Al examen neurológico presentó trastorno del sensorio, rigidez de nuca, ptosis palpebral completa derecha, midriasis derecha, lenguaje y atención alterados, compromiso del III, IV y VI pares craneales derechos, fotofobia y paresia de miembros superiores. Se planteó como diagnóstico una meningoencefalitis tuberculosa (MEC-TBC).

Se investigó que 10 meses antes, inició dolor en hemicadera izquierda. Cinco meses antes, el cuadro progresó hasta limitar la deambulación, haciéndose necesario el uso de muletas. Inició automedicación de antiinflamatorios no esteroideos y dexametasona diaria por vía oral durante cinco meses, notando acentuada ganancia ponderal. Un mes antes del ingreso a emergencias fue evaluado por el servicio de traumatología con el diagnóstico de coxoartritis izquierda.

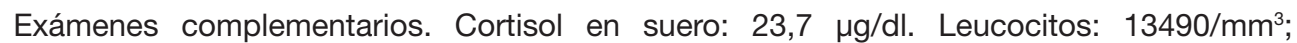
hemoglobina $14,70 \mathrm{~g} / \mathrm{dl}$; plaquetas $360000 / \mathrm{mm}^{3}$.

Hallazgos en LCR: aspecto cristal de roca; células $5 \times C$; $100 \%$ linfocitos; proteínas: $215 \mathrm{mg} /$ dl; glucosa: 29,3 mg/dl; ADA: 5,89 U/L. Tinta china: negativo. GRAM: no patológico. Método Gene-Xpert para BK en LCR positivo. Bacilos ácido-alcohol resistente en LCR: negativo. Baciloscopía en LCR y esputo negativos.

En la figura 1 se presenta la radiografía de tórax que no tiene hallazgos patológicos. 


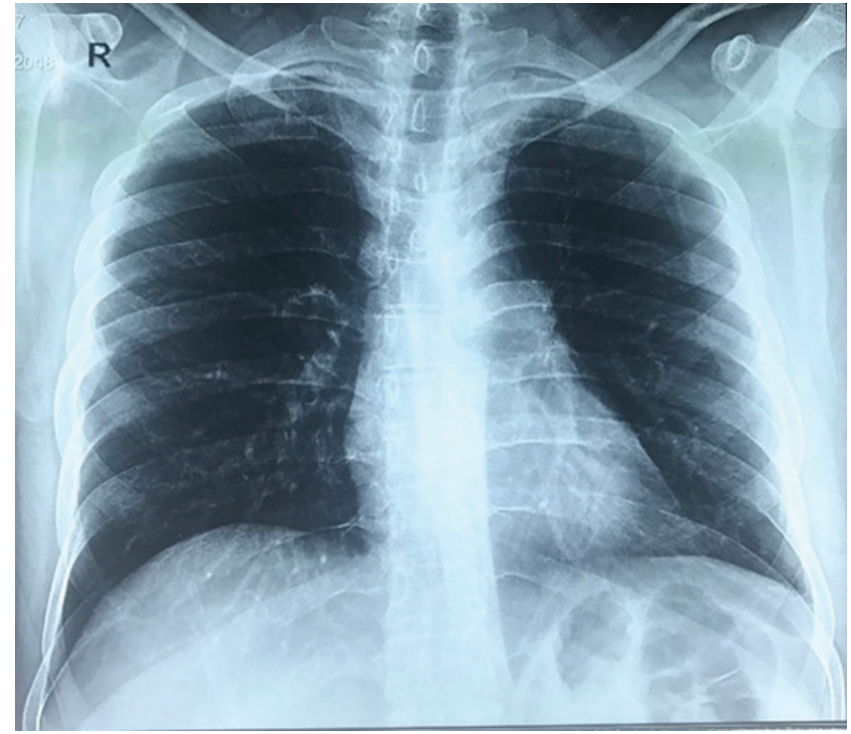

En la figura 2A la Resonancia magnética (RM) de pelvis al ingreso donde se evidencia un absceso en zona lateral a cabeza de fémur izquierdo. En la figura 2B la radiografía de pelvis al mes del tratamiento donde se aprecia erosiones subcondrales a nivel de la cabeza del fémur izquierdo, severa disminución del espacio coxofemoral y extenso patrón permeativo mal definido a nivel de la diáfisis que incluye la línea intertrocantérea.

Figura 2: Fig. 2A. RM cadera. Fig. 2B. Radiografía de pelvis al mes de tratamiento.

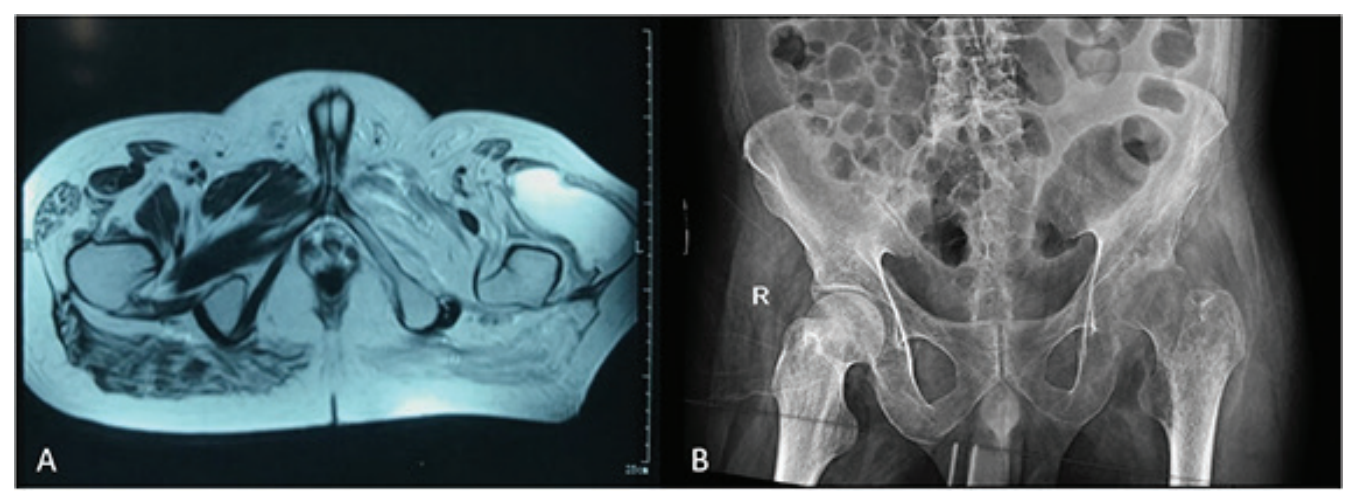

En la figura 3 A y B se presenta RM encefálica que muestra un tuberculoma, edema en lóbulo parietal derecho y realce meníngeo. En figura 3C RM encefálica en secuencia Flair que muestra alta señal en la sustancia blanca subcortical del lóbulo parietal ascendente y leptomeninge, que refuerzan de forma granular, en anillo. En figura 3D TC cráneo que muestra tuberculoma y edema en base del cráneo, causando disminución del ventrículo derecho por efecto de masa. 


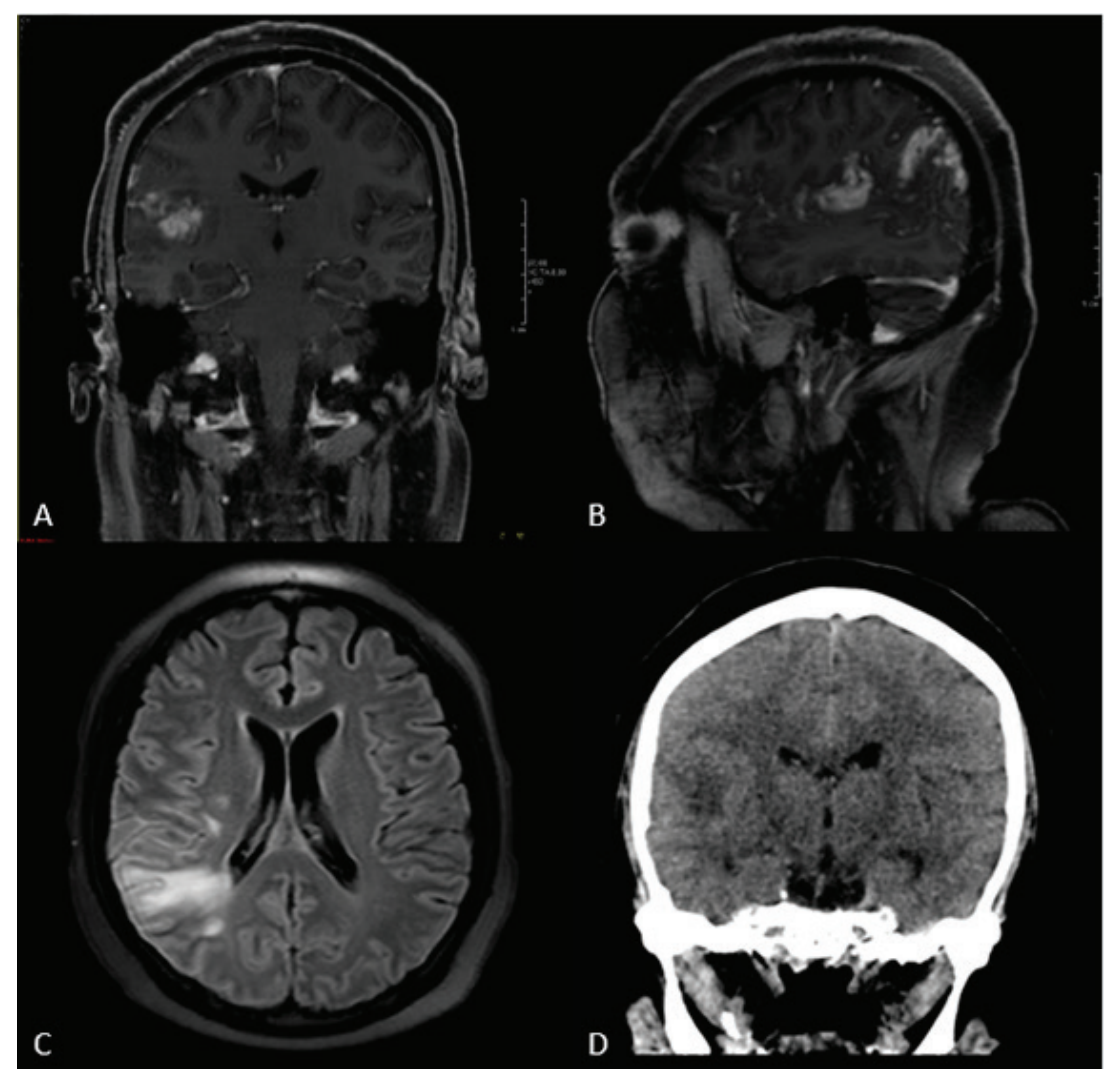

Figura 3: Figs. 3A y 3B: RM encefálico.

Punción aspirado con guía ecográfica del absceso peritrocantéreo izquierdo positiva a la baciloscopía, diagnosticando coxoartritis tuberculosa (CA-TBC).

Se realiza tratamiento con esquema antituberculoso: dos meses de etambutol $(20 \mathrm{mg} / \mathrm{kg})$, isoniacida (10 mg/kg), pirazinamida $(35 \mathrm{mg} / \mathrm{kg})$ y rifampicina $(15 \mathrm{mg} / \mathrm{kg})$ diarios, y diez meses siguientes con isoniacida $(10 \mathrm{mg} / \mathrm{kg}$ ) y rifampicina $(15 \mathrm{mg} / \mathrm{kg})$ tres veces semanales; tramadol (100 mg/día), dimenhidrinato (50 mg/día) y dexametasona (8 mg/día).

Paciente continúa hospitalizado con evolución favorable desde el punto de vista neurológico. Presenta episodios de fiebre leve. Se le llegó a inmovilizar ambos miembros inferiores con yeso desde la zona abdominal baja hasta el tobillo en el miembro inferior izquierdo y zona suprapatelar en el miembro inferior derecho, buscándose generar una anquilosis, para posteriormente evaluar la posibilidad de colocar una prótesis.

\section{Discusión}

Se reporta un caso de MEC-TBC en un adulto seronegativo sin antecedentes sugestivos de TBC pulmonar.

Este paciente no presentó manifestaciones radiológicas que evidencien una TBC pulmonar primaria: consolidación, tuberculomas, cavitaciones, entre otras ${ }^{(10)}$. Debido al contacto con diferentes grupos poblacionales en los viajes interprovinciales no se puede descartar que el foco primario sea pulmonar silente ${ }^{(9)}$, de no ser el caso, se plantea la inoculación directa. El consumo diario inicial de glucocorticoides explica la causa exógena del síndrome de Cushing y la inmunosupresión que facilitó la posibilidad de la diseminación del bacilo desde la cadera hasta el SNC.

El BK que infecta el SNC tiene tres formas de presentación clínica: meningitis, tuberculoma intracraneal y aracnoiditis espinal(11).

Se describen cuatro hallazgos en MTBC: realce meníngeo basal, tuberculomas intracraneales, hidrocefalia e infartos ${ }^{(3)}$, evidenciándose los dos primeros en el paciente.

El realce meníngeo (Figura 3A y 3B) se explica por el proceso infeccioso a nivel mesencefálico (4), lo que evidenciaría la afectación del descenso de los axones motores, explicando la paresia. El hallazgo de tuberculomas en lóbulo parietal derecho y el edema en base del cráneo (Figura 3) 
explican la alteración del sensorio y la compresión de la salida de los pares craneales III, IV y VI. Esta última es sugestiva de MTBC debido al compromiso de la base craneal(12).

Los tuberculomas observados en el lóbulo parietal derecho generaron un edema que afectó las áreas de Wernicke y del lenguaje (Figura 3), asimismo, la focalización neurológica es evidencia semiológica de una tumoración cerebral, cuyo diagnóstico etiológico abarca a los tuberculomas. La encefalitis se evidenció clínicamente a partir de la afectación del sensorio, parálisis de pares craneales, vómitos; y la meningitis, por la rigidez de nuca y fotofobia.

Entre los hallazgos del LCR, destaca el aspecto en "cristal de roca", propio de MTBC y meningitis viral. Se resalta que en la meningitis se suele hallar un aumento en el recuento celular, lo cual no se observa en este caso, ya que solo presenta 5 células por campo debido a la inmunosupresión, sin embargo, la hipoglucorraquia e hiperproteinorraquia son características de la meningitis bacteriana y MTBC.

El ADA (+) es un marcador rápido de MTBC. El 100\% de linfocitos orienta el diagnóstico hacia una MTBC, mientras que en una meningitis bacteriana se suele hallar un predominio de polimorfonucleares ${ }^{(13)}$.

Adicionalmente, la disociación albúmino-citológica (elevación de proteínas con recuento celular normal) es un indicador sensible de MTBC.

Sobre la TBCOA, la RM evidencia coexistencia granular y exudativa, pero el absceso indica predominancia del último (Figura 2A), por otro lado, el patrón permeativo de la radiografía (Figura 2B) determina la evolución en la destrucción de la cabeza del fémur.

La corticoterapia que recibió el paciente durante 5 meses repercutió notablemente sobre su estado inmunitario. Se ha descrito que el uso prolongado de corticoesteroides aumenta la apoptosis de las células T y disminuye la producción de interferón - $\gamma$. Sin embargo, estudios recientes señalan que los macrófagos también se verían afectados, inhibiéndose la respuesta inmune innata, su producción del óxido nítrico, como antimicrobiano, y la autofagia, por lo cual la supervivencia intracelular del BK se vería aumentada ${ }^{(14)}$.

El abuso de corticoides causa severas complicaciones, sin embargo, empleados correctamente mejoran el cuadro clínico de MEC-TBC en etapas iniciales ${ }^{\left({ }^{10}\right)}$. Según Leonard J. (10), la TBC del SNC puede tratarse con dexametasona en una dosis de 12mg/día para un adulto aproximadamente. Teniendo esto como referencia, el plan terapéutico incluyó dexametasona endovenosa (8mg/día).

La inmovilización mediante el uso de yeso se da en periodos desde un mes hasta tres meses dependiendo de la severidad del caso. No se ha llegado a reportar el porcentaje de éxito de esta terapia.

El presente es un caso de diseminación tuberculosa que asocia MEC-TBC a CA-TBC por migración del BK desde la articulación coxofemoral hasta SNC en un paciente adulto y seronegativo, siendo estas condiciones infrecuentes epidemiológicamente, ya que del total de casos de TBCOA, la columna representa el $40 \%$; la cadera el $12 \%$; y la rodilla del 7 al $8 \%$, por lo que se considera importante divulgar este caso para ampliar la experiencia médico-científica.

Dentro de las limitaciones, la TBCOA no fue diagnosticada mediante biopsia de la membrana sinovial ni aspirado del líquido articular, además únicamente se inmovilizó los miembros afectados, y el paciente quedó en espera de una prótesis, sin embargo, los únicos antibióticos administrados fueron los del esquema antituberculoso. Tampoco se contó con un cultivo de LCR para BK ni se evidenció al bacilo en examen directo, debido a la escasez de este en la zona lumbar del espacio subaracnoideo ${ }^{(15)}$. No obstante, actualmente el diagnóstico de tuberculosis del sistema nervioso central se apoya en las técnicas moleculares, como Gene-Xpert (positiva en este paciente), mientras que los demás estudios suelen tener rendimientos (especificidad y sensibilidad) variables y dependientes del grado de inmunosupresión del paciente, por lo que el método usado fue adecuado para la situación clínica del paciente. También se evidenció la meningoencefalitis tuberculosa mediante la clínica y la respuesta favorable al tratamiento antituberculoso.

\section{Conflicto de intereses}

Los autores declaran no tener conflicto de intereses. 


\section{Agradecimientos}

A la Dra. Guillermina Ballena Effio por el informe de las imágenes radiológicas mostradas en este caso clínico y por la realización de la punción aspirado con guía ecográfica.

\section{Bibliografía}

1- Agenda Nacional de Investigación en Tuberculosis en Perú, 2011-2014. Rev Panam Salud Pública. 2013; 33(2): 151-158

2- Perú. Ministerio de Salud Pública. Análisis de la situación epidemiológica de la tuberculosis en el Perú 2015. Lima: MINSA; 2016.

3- Saavedra J, Urrego S, Pérez A, Eugenia M. Diagnóstico de meningitis tuberculosa. Acta Neurol Colomb. 2015; 31 (2): 223-230

4- Mera J, Granados A, Toro J, Delgado D, González A. Caracterización del realce meníngeo intracraneal. Rev. Colomb. Radiol. 2017; 28(3): 4709-16

5- Bejarano N, Zarallo L, Cardesa J. Meningitis tuberculosa. Revisión epidemiológica y clínica de los pacientes ingresados en el hospital M-Infantil de Badajoz, durante los últimos 15 años. Vox Paedriatrica. 2008; 16(1): 14-17.

6- 6Meningitis tuberculosa: claves para su diagnóstico y propuestas terapéuticas. Rev Chil Infect 2011; 28 (3): $238-247$.

7- Kramer N, Rosenstein E. Rheumatologic manifestations of tuberculosis. Bull Rheum Dis. 1997; 46(3):58.

8- Villafañe M, Corti M, Palmieri O, Castro M. Tuberculosis de la cadera. Presentación de un caso y revisión de la literatura. Rev chil infectol. 2015; 32(5):580-583.

9- Ruiz-Ozuna y González-Colunga. Tuberculosis osteoarticularcaseificante de cadera con destrucción articular grave: presentación de un caso. Acta ortop. mex. 2008; 22(4): 254-258.

10- Leonard J. Central Nervous System Tuberculosis. Microbiol Spectr. 2017; 5(2): 1-10.

11- Castiñeira Estévez A, López Pedreira MR, Pena Rodríguez MJ, Liñares Iglesias M. Manifestaciones radiológicas de la tuberculosis pulmonar. Med Integr. 2002; 39(5):192-206.

12- Reuter H, Wood R, Schaaf HS, Donald PR. Overview of extrapulmonary tuberculosis in adults and children. In: Zumla Al, Schaaf HS. ed. Tuberculosis a comprehensive clinical reference. Saunders: Philadelphia; 2009. pp. 377-390.

13- Lobo J. Meningitis bacteriana y viral. Med. Leg Costa. 2016; 33(1): 234-245.

14- Wang J, Wang R, Wang H, Yang X, Yang J, Xiong W, et al. Glucocorticoids Suppress Antimicrobial Autophagy and Nitric Oxide Production and Facilitate Mycobacterial Survival in Macrophages. Sci Rep. 2017 Apr 20;7(1):982.

15- Morales-Aguirre J. Infección por micobacterias del sistema nervioso central. Bol Med Hosp Infant Mex. 2006; 23: 332-350.

\section{Aporte cada autor al trabajo}

Marcia Darmelly Salas Pérez: concepción y diseño del trabajo, recolección de datos, análisis e interpretación de los datos, revisión crítica del manuscrito.

Oscar Orlando Rivera Torrejón: concepción y diseño del trabajo, recolección de datos, análisis e interpretación de los datos, redacción del manuscrito, revisión crítica del manuscrito.

Diego Chambergo Michilot: concepción y diseño del trabajo, recolección de datos, análisis e interpretación de los datos, redacción del manuscrito, revisión crítica del manuscrito.

Ubaldo Paucar Pongo: concepción y diseño del trabajo, recolección de datos, análisis e interpretación de los datos, redacción del manuscrito, revisión crítica del manuscrito. 


\section{Notas}

TBC: tuberculosis

SNC: sistema nervioso central

MTBC: meningitis tuberculosa

LCR: líquido cefalorraquídeo

ADA: adenosíndesaminasa

TBCOA: tuberculosis osteoarticular

MEC-TBC: meningoencefalitis tuberculosa

RM: resonancia magnética

CA-TBC: coxiartritis tuberculosa 